A. Michelsen · K.-G. Heller · A. Stumpner · K. Rohrseitz

\title{
A new biophysical method to determine the gain of the acoustic trachea in bushcrickets
}

Accepted: 4 March 1994

\begin{abstract}
A method is described for measuring the gain (i.e., the change of amplitude and phase angle) for sounds that propagate to the internal surface of the tympana in ears working as pressure difference receivers. The gain of the acoustic trachea has been measured in two similarly sized and closely related species of bushcrickets, in which the acoustic spiracles and tracheae differ markedly in size. The amplitude part of the gain is much larger in the species with the larger acoustic spiracle, whereas the phase part is very similar in the two species. The method is compared with other methods, which in the past have been used for estimating the gain of sound pathways inside animal bodies.
\end{abstract}

Key words Hearing - Pressure difference receiver Bushcricket

\section{Introduction}

The ears of bushcrickets are located in the tibiae of the forelegs. Each ear has two tympana which are formed from thin regions of leg cuticle in contact with the walls of the modified leg trachea (review: Bailey 1990). The proximal end of this acoustic trachea opens on the lateral side of the prothorax. The size of the trachea and its opening vary considerably between different species of bushcrickets. In most bushcrickets the access of sound to the external surface of the tympana is largely restricted by cuticular folds that leave only narrow "tympanal slits" communicating with the exterior. In other bushcrickets such folds are absent.

\footnotetext{
A. Michelsen ( $\forall)$ - K. Rohrseitz

Centre for Sound Communication, Institute of Biology,

Odense University, DK-5230 Odense M, Denmark

\section{K.-G. Heller}

Institute of Zoology, Erlangen University,

Staudtstrasse 5, D-91058 Erlangen, Germany

\section{A. Stumpner}

Institute of Zoology I, Göttingen University,

Berliner Strasse 28, D-37073 Göttingen, Germany
}

The responses of most bushcricket ears vary significantly with the angle of sound incidence, but the reason for this directionality has been much debated. Five different mechanisms have been suggested: (1) The sounds acting at the external and internal surfaces of the tympana have roughly similar amplitudes, and the ear has the directionality of a pressure difference receiver; (2) The sound pressure at the internal surface is much larger than that at the external surface, and the directionality is due to sound diffraction by the body; (3) As the second suggestion, but the directionality is that of the horn-shaped acoustic trachea; (4) The sound pressure at the external surface dominates, and the directionality is caused by diffraction of sound around the leg; (5) As the fourth suggestion, but the diffraction occurs at the entry of the tympanal slits.

Extensive research in the late 1970's and early 1980's (review: Lewis 1983) provided much evidence in favour of the second of these mechanisms, but a thorough physical analysis was not possible with the techniques then available. It was therefore not possible to sort out which of the different findings were caused by genuine differences between the species studied, and which were due to the very different methods used by the investigators. Recently, evidence has been obtained for the notion that different species of bushcrickets may exploit different physical mechanisms (pressure or pressure gradients) for their directional hearing (e.g., Mason et al. 1991, Stumpner and Heller 1992).

The present investigation was inspired by new technology (very powerful computers and a probe microphone with a tip diameter of $1 \mathrm{~mm}$ and a good sensitivity at ultrasonic frequencies). Furthermore, we had access to two similarly sized species of bushcrickets without cuticular folds and tympanal slits (thus excluding the fifth mechanism) and with acoustic spiracles and tracheae of very different sizes. We present a new method which allows a direct determination of the gain of the acoustic trachea. We find that the gain of the acoustic trachea is much larger in the species with the larger spiracle. The results are in agreement with the first and the second of the mechanisms mentioned above. 


\section{Materials and methods}

Two species of bushcrickets of the genus Poecilimon, $P$. thessalicus ssp. veluchianus Ramme and $P$. laevissimus Fischer were collected in Greece (see Stumpner and Heller 1992) and kept in the laboratory. The experiments were performed in Odense in June 1991. The animals were briefly anaesthetised and mounted dorsal side up on a piece of cork at the end of a thin metal rod fastened to a heavy stand.

The vibration velocity of one of the tympana was measured with a laser vibrometer (Dantec). The vibration amplitude varies with the position on the tympanum, and a very small, highly reflecting sphere (weight approx. $1 / 2 \mu \mathrm{g}$, from $3 \mathrm{M}$ reflective tape) was therefore placed on the tympanum, thus allowing us to locate the same spot on the membrane after experimental manipulations of the preparation. The presence of the sphere did not affect the frequency response of the tympanum (Stumpner and Heller 1992).

An FFT-analyzer (Hewlett Packard 3562A) generated brief (12 $\mathrm{ms})$ frequency sweeps $(0-50 \mathrm{kHz})$ for the loudspeakers, and it calculated transfer functions between the various signals, e.g., those received from the laser vibrometer and microphones. In most of the experiments, a specially designed near-field sound source was used (see below). In other experiments, a tweeter (Dynaudio D28AF) was placed $40 \mathrm{~cm}$ from the preparation.

A probe microphone (Brüel and Kjær 4182) was used for monitoring the sounds less than one mm from the tympanum or from the entrance of the acoustic trachea. It had a tip diameter of $1 \mathrm{~mm}$, a smooth amplitude and phase response, and a reasonable sensitivity up to at least $100 \mathrm{kHz}$ (it is as sensitive as the $1 / 8$ “ $\mathrm{B} \& \mathrm{~K}$ microphone 4138 at $10-20 \mathrm{kHz}$; at $50 \mathrm{kHz}$ and $100 \mathrm{kHz}$ its sensitivity is approximately 20 and $40 \mathrm{~dB}$ less, respectively). The input impedance of the probe as determined by Brüel and Kjær was sufficiently high for these measurements $\left(8 \times 10^{8} \mathrm{Ns} / \mathrm{m}^{5}\right)$.

The probe microphone was calibrated in a free sound field by comparing its response to that of a $1 / 8$ inch microphone (Brüel and $\mathrm{Kjær} 4138$ ). The result of this calibration (an amplitude and a phase curve) was used for correcting the sound spectra obtained with the probe microphone. This was easily done with the signal analyzer, but the correction added noise to the measured data. This could be avoided by performing the correction with a smoothed version of the amplitude and phase curves (the analyzer fitted the measured data to a mathematical function, which was then used for calculating smooth curves).

\section{The local sound source}

For the experiments we needed a local sound source, which would allow us to apply sound to one input at a time (tympanum or spiracle). This was obtained by guiding sound from a tweeter (Onkyo TW-3300A ring radiator), through $30 \mathrm{~cm}$ of plastic tubing, to a glass pipette. Various sizes of pipette tips were tested, and smal lumps of glass wool were needed at various places in the sound guide in order to reduce tube resonances. The frequency response is shown in Fig. $2 \mathrm{~A}$.

The pipette used had an internal tip diameter of $2.5 \mathrm{~mm}$. The amplitude of the sound pressure was at a maximum within a few $\mathrm{mm}$ from the tip and $15-20 \mathrm{~dB}$ less at a distance of $2 \mathrm{~cm}$. The phase of the sound pressure changed as expected from the distance to the tip and evenly in all directions.

The pipette tip obviously behaved as a small monopole sound source of simple geometry. Very close to sound sources (in the near field) the air particle velocity is much larger than at a similar sound pressure in the far field. It was therefore necessary to test whether this near field source activated the hearing organ in the same manner as far field sources (see Discussion).

\section{Determination of the gain of the acoustic trachea}

The gain $(G)$ of the acoustic trachea is the frequency dependent change in amplitude and phase angle of sounds propagating from

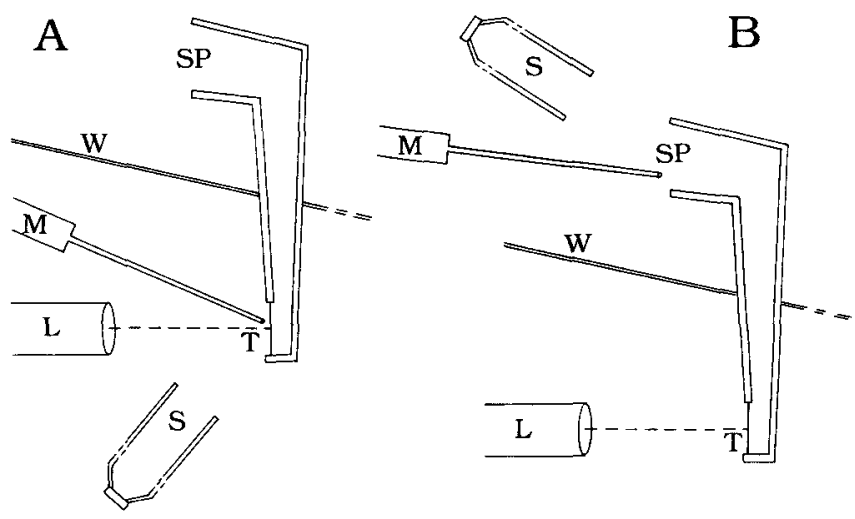

Fig. 1A,B Measuring the gain of a transmission path to the internal surface of the tympanum (T). A The tympanal membrane is driven by sound at its external surface. $\mathbf{B}$ The tympanal membrane is driven by sound arriving at its internal surface from the spiracular opening $(S P)$ of the transmission path. $L$ laser vibrometer; $M$ probe microphone; $S$ local sound source; $W$ wall made of bees' wax. Dimensions not to scale. Further explanation in the text

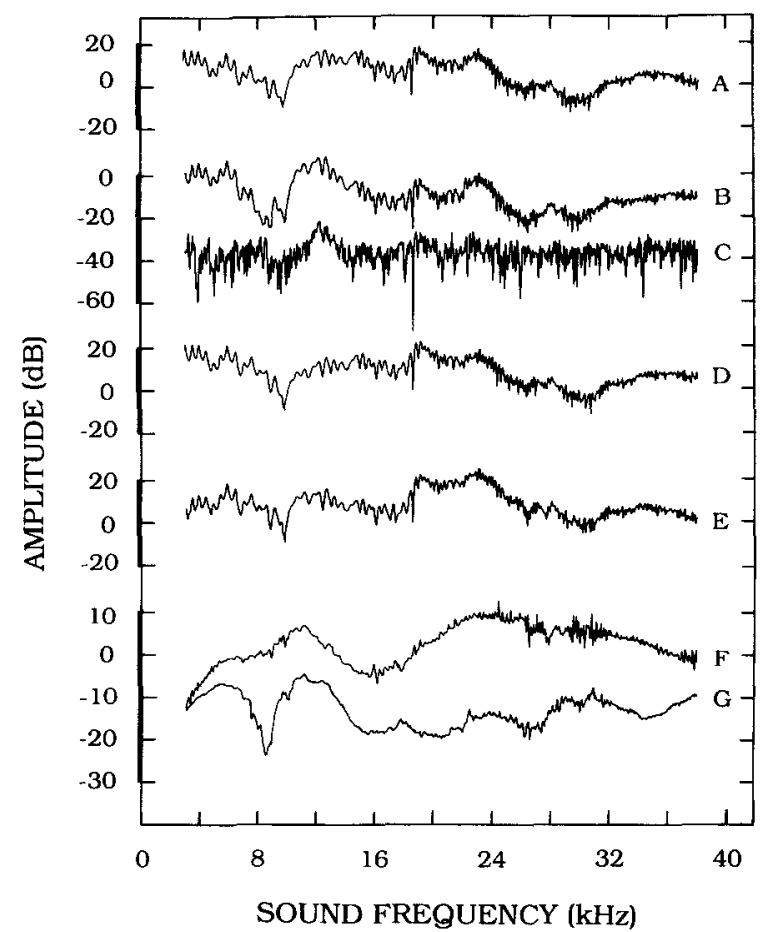

Fig. 2 Amplitude spectra obtained during the determination of the gain of an acoustic trachea in $P$. laevissimus. $A, B, D$ and $E$. Sound pressures $(A$ and $D)$ and tympanic vibration velocities $(B$ and $E$ ) measured with the local sound source and the probe microphone close to the tympanum ( $A$ and $B$, see Fig. 1A) and close to the spiracle ( $D$ and E, see Fig. 1B), respectively. $F$ and $G$. Transfer functions (i.e., the vibration velocity at a constant sound pressure) obtained by division $(F=E / D$ and $G=B / A)$. C. Vibration velocity of the leg cuticle close to the tympanum during the determination of $B$. In $A$ and $D,-9 \mathrm{~dB}$ corresponds to $1 \mathrm{~Pa}$. In $B, C$ and $E, 0 \mathrm{~dB}$ corresponds to $2.21 \mathrm{~mm} / \mathrm{s}$ (rms). In $F$ and $G, 0 \mathrm{~dB}$ corresponds to $0.79 \mathrm{~mm} / \mathrm{s}(\mathrm{rms})$ at $1 \mathrm{~Pa}$. Further explanation in the text

the spiracular opening to the internal surface of the tympanum. The sound pressure at the spiracle can be measured directly with the $\mathrm{B} \& \mathrm{~K}-4182$ probe microphone. The result is quite reliable at frequencies where the probe does not cause much diffraction (be- 
low $35 \mathrm{kHz}$ for a tip of $1 \mathrm{~mm}$ diameter). However, the probe microphone can not measure the true sound pressure at the internal surface of the tympanum (see Discussion).

Our approach has been to measure the sound pressure behind the tympanum indirectly by using the tympanum as a microphone and monitoring its vibrations with laser vibrometry. The experiment was divided into two main steps. First, we "calibrated" our "microphone" by applying sound exclusively to its external surface (Fig. 1A). Then we observed the tympanal vibrations in a situation, where the tympanum was driven from its internal surface by sound which arrived exclusively from the spiracular input (Fig. $1 B$ ). In each case, the sound pressure was measured with the probe microphone. A wall of bees' wax between the auditory inputs (and the fact that the sound pressure decreases rapidly with the distance to the local sound source) ensured that the sound pressure was very low at the "shaded" input (this was confirmed by moving the tip of the probe microphone).

In each step of the experiment, the effect of the sound on the tympanal vibration was quantified by calculating the transfer function. The transfer function is the tympanal velocity divided by the sound pressure; it has both amplitude and phase components. In the first step (Fig. 1A), the transfer function (A) was that of the tympanum. In the second step (Fig. 1B), the transfer function (B) was the gain of the transmission path multiplied by the transfer function of the tympanum. By dividing $B$ with $A$ we were left with the gain function.

With this approach we made the assumption that the impedance of the tympanum does not depend on whether the tympanum is caused to oscillate by sounds at the outer surface or by sounds acting at the inner surface. We do not know, whether this is true. If not, then the difference is included in the measured transmission gains. This uncertainty does not affect the considerations of the mechanisms responsible for directional hearing. The method also rests upon the assumption that the hearing organ reacts in the same manner to the local sound source as to normal far field sound sources, i.e. that the sound pressure measured by the probe was in fact the effective stimulus. This was confirmed in control experiments (see Discussion).

When interpreting the phase part of the gain it should be noted that the method for estimating the gain adds $180^{\circ}$ to the real values (the vibrations of the tympanum are used to disclose the pressure at its internal surface, but the "calibration" of the tympanum is performed with a sound pressure at its external surface).

The full procedure can be illustrated with data from an experiment on a specimen of $P$. laevissimus (Fig. 2 , which shows only the amplitude parts of the spectra). The sound spectra (A and D) were quite noisy, and the vibration spectra (B and $E$ ) of course showed a similar number of maxima and minima. The transfer functions ( $F$ and $G$ ), however, were smoother. The vertical distance between $F$ and $G$ is the amplitude part of the gain of the acoustic trachea (which is plotted in the upper part of Fig. 3). Some sources of error are considered in the Discussion.

\section{Results}

The gain of the acoustic trachea measured in the specimen of $P$. laevissimus, which provided data for Fig. 2, is shown in Fig. 3. The amplitude part of the gain increases from c. $0 \mathrm{~dB}$ (a gain of 1 , that is no change of amplitude) at low frequencies to $10-25 \mathrm{~dB}$ (a 3-18 times increase in amplitude) between 8 and $40 \mathrm{kHz}$.

At low frequencies, the phase angle is close to $180^{\circ}$, which means that the sound propagates through the acoustic trachea with only little phase shift (see Methods).

The phase changes c. $720^{\circ}$ from 2 to $40 \mathrm{kHz}$. In other words, a $40 \mathrm{kHz}$ sound experiences a phase shift of ca. two cycles. Two cycles at $40 \mathrm{kHz}$ have a duration of 50
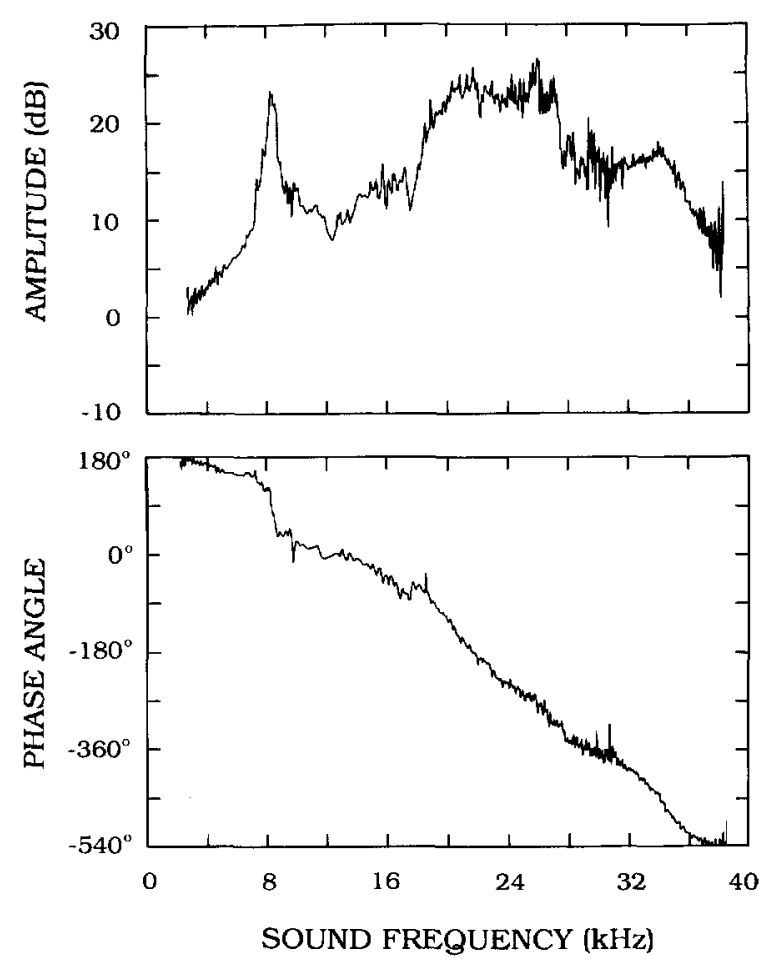

Fig. 3 The gain of the acoustic trachea in the specimen of $P$. laevissimus from Fig. 2. The maximum at $8-9 \mathrm{kHz}$ is probably an artifact (see Discussion). The phase part of the gain is a measure of how much the sound is delayed by propagating through the acoustic trachea (see text)
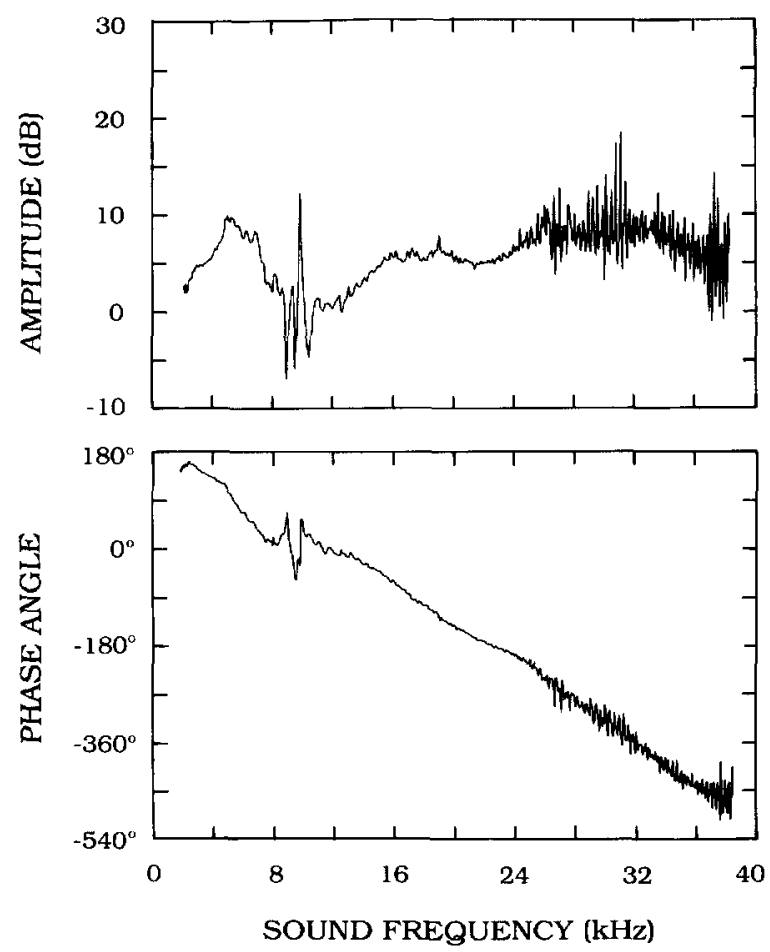

Fig. 4 The gain of the acoustic trachea in a specimen of $P$. thessalicus. The large oscillation around $10 \mathrm{kHz}$ is an artifact 


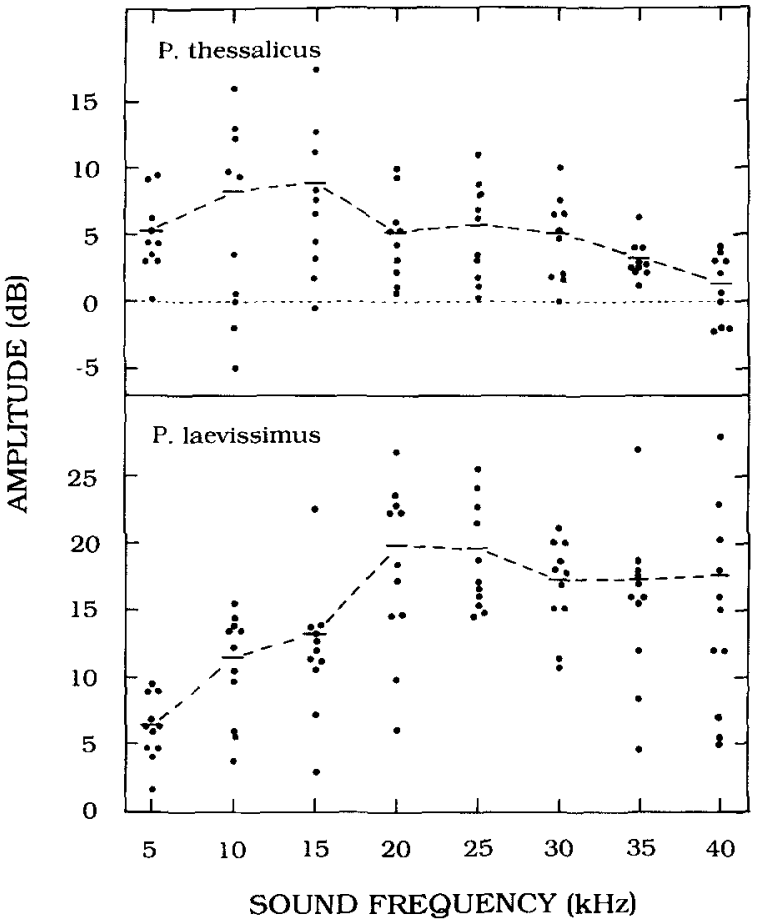

Fig. 5 The average amplitude gain observed in the two species within eight $5-\mathrm{kHz}$ frequency bands centered at the frequencies indicated ( $P$. thessalicus: 10 measurements from 5 animals; $P$. laevissimus: 11 measurements from 6 animals). The average values are connected by dashed lines

$\mu \mathrm{s}$, which is the time that it takes for the sound to propagate through the acoustic trachea. The phase is almost linearly related to frequency, so the propagation velocity of the sound is likely to be independent of frequency. Some small deviations from a linear relationship correspond to maxima or minima in the amplitude part of the gain.

The gain measured in a specimen of $P$. thessalicus (Fig. 4) follows the pattern just described, but the amplitudes obviously are much smaller than those in Fig. 3.

The bodies and tympana of the two species of Poecilimon investigated are of similar size, but the spiracular entrance of the acoustic trachea is ca. 4 times larger in $P$. laevissimus than in P. thessalicus (Stumpner and Heller 1992). This difference is clearly reflected in the amplitude gain of the acoustic tracheae.

These animals are very delicate, and vibrations in their long, thin legs tend to be of much larger amplitude than in the legs of, e.g., crickets. The data in Figs. 3 and 4 are therefore fairly noisy, and they also include some artifacts (which are considered in the Discussion). The data from several other experiments were even more noisy. A very coarse smoothing of the data was performed by reducing the frequency resolution to eight $5-\mathrm{kHz}$ frequency bands centered by the frequencies $5,10,15$, $40 \mathrm{kHz}$ and indicating the average level at each frequency band. In these smoothed data (Fig. 5), the difference in the amplitude gain in the two species appears to be especially prominent at $20-30 \mathrm{kHz}$, the frequency band of the calling songs. The data show a considerable scatter, which appears to reflect large inter-animal variability (the variability between the gains obtained in repeated determinations in single animals was much lower).

\section{Discussion}

Controls of the method for determining the gain

One of the assumptions behind the method for determining the gain of the acoustic trachea is that the two auditory inputs (tympanum and spiracle) respond exclusively to the pressure component of sound, which is measured by the probe microphone. In other words, it is assumed that the local (near-field) source can substitute the normal far-field sources. In one kind of test, the transfer functions were calculated between the tympanal vibrations and the sound pressure at the entrance of the acoustic trachea in intact $P$. laevissimus activated with sound from the near-field source and with sound from a loudspeaker $40 \mathrm{~cm}$ away. Small differences were observed in individual tests (Fig. 6A), but they were not systematic at frequencies where the tracheal gain was much larger than one (a situation where the effect of the sound acting at the external surface of the tympanum can be ignored).

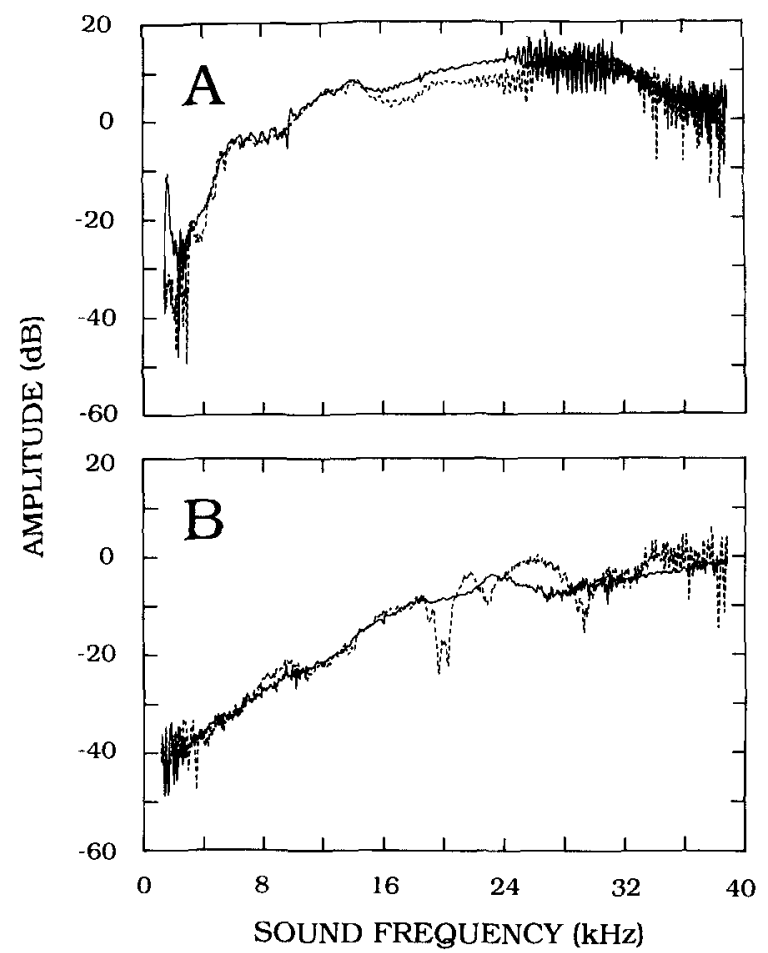

Fig. 6A,B Control measurements in $P$. laevissimus comparing the transfer functions (tympanic vibrations at a constant sound pressure) obtained with a local sound source (solid line) and sound from a loudspeaker $40 \mathrm{~cm}$ away (dashed line). Sound applied to and measured at: A the acoustic spiracle; B the external surface of the tympanum (the acoustic spiracle was closed by wax). (These recordings have not been corrected for the characteristics of the probe microphone, so the shape of the curves differ from those in Fig. 2) 
In another kind of test the same procedure was applied at the front surface of a tympanum in animals, in which the entrance of the acoustic trachea had been blocked with bees' wax (Fig. 6B). Again, no systematic differences were detected between the transfer functions obtained with the near- and far-field sources.

Both kinds of tests thus demonstrated that the nearfield source faithfully mimicked the far-field source, and that only the pressure component of sound is important at the acoustic inputs of this hearing organ. Similar results were obtained in crickets. However, a word of warning is in order. Such tests must be performed, if this technique should be used in other preparations.

\section{Some sources of error in the determination of the gain}

The method described here can produce data for both the amplitude and phase of the gain of auditory pathways. It should be noted, however, that several sources of error have to be considered. One problem is that the gain is computed from four primary measurements (frequency spectra), each contributing noise to the final estimate of the gain. This is, however, generally a minor problem.

In the estimation of the amplitude of the gain it is assumed that the probe microphone measures the exact sound level at the auditory input(s). This may not be the case, if the local sound source is placed very close to the microphone tip and the auditory inputs (since the sound level changes rapidly with the distance, when one approaches a sound source). In this situation, it is not so difficult to measure the physical position of the objects. The problem is that one cannot be sure of the position of the acoustic centre for the auditory inputs (acoustic receivers respond to the sound pressure at a point in space, the acoustic centre, the position of which is determined by diffraction; it is generally not exactly at the structure itself). A systematic error of several $\mathrm{dB}$ in the estimate of the amplitude part of the gain may be the result of too small a distance to the sound source. The distance was therefore several $\mathrm{mm}$ in our experiments.

In the present experiments, the main source of error was due to the sound-induced vibrations in the thin legs carrying the ears. This problem can be illustrated by the following example:

In Fig. 2, curves $B$ and $C$ show the vibration velocities of the tympanum and of the cuticle of the leg next to the tympanum, respectively. At most frequencies, B is at least $20 \mathrm{~dB}$ above $\mathrm{C}$, but between 8 and $9 \mathrm{kHz}$ the difference is only $4-5 \mathrm{~dB}$, and here we observe a large minimum in $G$ (which is B divided by A). Furthermore, the phase curves (not shown) suggest that the tympanum and this part of the leg were vibrating almost out of phase at $8-9 \mathrm{kHz}$. The laser vibrometer measures the velocity relative to itself (and other stationary objects), and the vibration measured from the tympanum may thus have been considerably smaller than the vibration of the tympanum relative to the leg.
Unfortunately, the beam-like vibrations of the legs are so complicated that one cannot use the data in $\mathrm{C}$ for correcting those in $\mathrm{B}$. In the amplitude part of the gain (upper curve in Fig. 3) the minimum in G has become a maximum (the gain is $F$ divided by $G$ ). These observations thus suggest that the large values at $8-9 \mathrm{kHz}$ should not be trusted.

The cure for this problem is to provide sufficient support (of bees' wax) for the thin legs, thus reducing their vibrations to an acceptable level. This is fairly easy in crickets (see the two consecutive papers), but not in the bushcrickets studied here.

\section{The acoustics of the bushcricket ear}

The amplitude of the gain of the acoustic trachea observed in the two species of Poecilimon is close to $1(0 \mathrm{~dB})$ at very low frequencies $(1-3 \mathrm{kHz})$, confirming earlier findings in other species of bushcrickets (Michelsen and Larsen 1978). At higher frequencies, the two species have very different gains. Both species produce calling songs with an intensity maximum at $20-30 \mathrm{kHz}$ (Stumpner and Heller 1992), where the species with the small spiracle ( $P$. thessalicus) has a gain of 5-6 dB, whereas the species with the large spiracle ( $P$. laevissimus) has a gain of 18-20 dB (Fig. 5). These results are in agreement with the notion of the acoustic trachea as a horn-shaped acoustic transformer, in which the gain is expected to depend on the size of the spiracle relative to the size of the trachea in the leg. It is interesting that Heinrich et al (1993) did not find any consistent differences in the amplitude gains measured from acoustic tracheae in seven species with a quite variable morphology of the acoustic trachea.

The phase part of the gain is very similar in the two species and almost linearly related to frequency (Figs. 3 and 4). This is the picture that one would expect in a simple delay-line, in which the propagation of the sound takes a certain time (here approximately $50 \mu \mathrm{s}$ ).

From the amplitude gains one may predict that, at the frequency band of the calling song, the ear of $P$. laevissimus is dominated by the sound arriving from the surface of the thorax, and that the sound acting at the external surface of the tympanum can be ignored. The directionality of the ear should thus be determined almost entirely by the diffraction around the thorax, which is, in fact, providing useful directional cues at $20-30 \mathrm{kHz}$ (Fig. 7A). In contrast, the much smaller gain in $P$. thessalicus indicates a much more mixed situation, in which both the internal and external sound pressures are important. Diffraction around the leg does not provide any useful directional cues at $20-30 \mathrm{kHz}$ (Fig. 7B), and the directional hearing of this species is thus likely to be inferior to that of $P$. laevissimus. These conclusions are in excellent agreement with the data obtained in these two species by Stumpner and Heller (1992, Fig. 4A), who studied how the tympanal vibrations and the sound pressures at the tympanum and spiracle depend on the direction of sound incidence. 
150

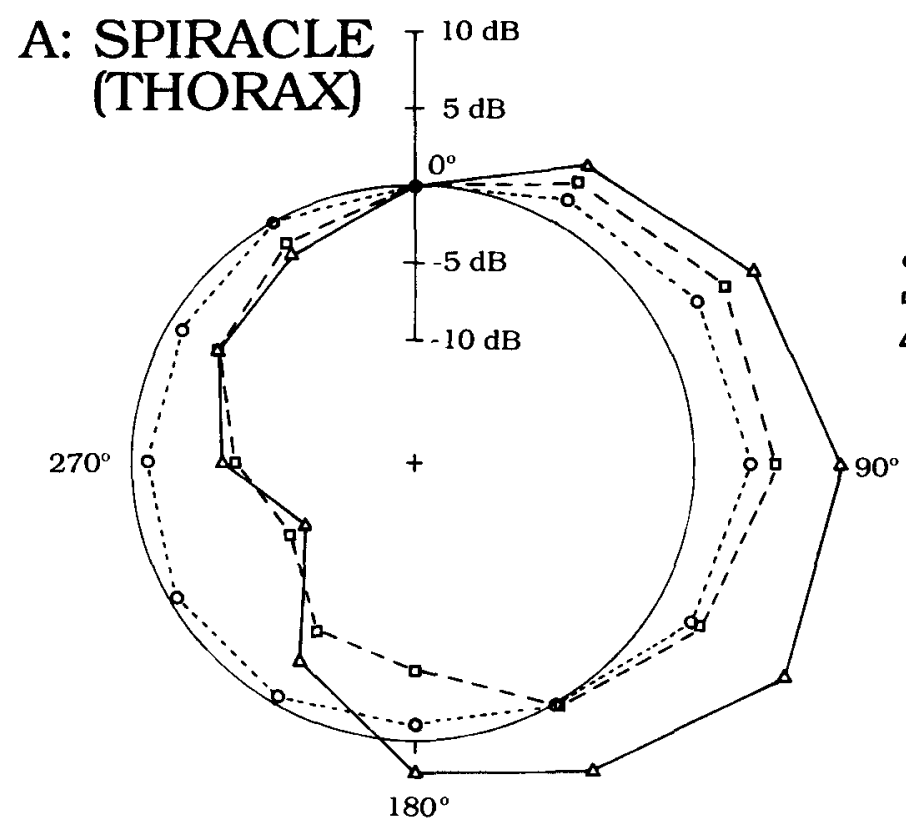

Fig. 7 Directional dependence of the sound pressure measured with the tip of the probe microphone at the entrance of the acoustic spiracle (A) and at the ear in one of the front legs (B) of a specimen of $P$. laevissimus. The sound pressures measured at various directions of sound incidence are plotted relative to the sound pressure measured when sound arrives from the forward $\left(0^{\circ}\right)$ direction. $90^{\circ}$ is ipsilateral. The animal was standing on ground at the centre of a roundabout carrying a loudspeaker (see Michelsen et al. 1994 for further details)

Earlier attempts at determining the gain of the acoustic trachea

\section{Blocking sound pathways}

Many investigators (including us) have blocked the entrance of the acoustic trachea and observed a large decrease in the sensitivity of the ear (measured as the neural threshold or the vibrations of the tympana). Similar blocking experiments have been performed in many other animals, and many investigators have tried to use the change of sensitivity for estimating the gain of the acoustic pathway. Such a procedure can be quite misleading.

From theory, we expect the velocity of the tympanal vibrations to be determined by two main factors: the force acting on the tympanum and the impedance of the tympanum. Both factors are likely to be changed by closing the acoustic trachea. The force changes, because sound is now acting only on the external surface of the tympanum. The impedance changes, because the tympanum's freedom to move is affected by its surroundings, e.g., the presence of an air space behind the tympanum. So, by blocking the entrance of the acoustic trachea we have simultaneously changed two parameters (and we have not learned much - it is similar to trying to find two unknown parameters from only one equation).

The change in the impedance of the tympanum by manipulations of its surroundings has, so far, only been expected from theory, but it can easily be measured. In Fig. 8 the change is shown in the vibration velocity of
A. Michelsen et al:: Gain of bushcricket acoustic trachea
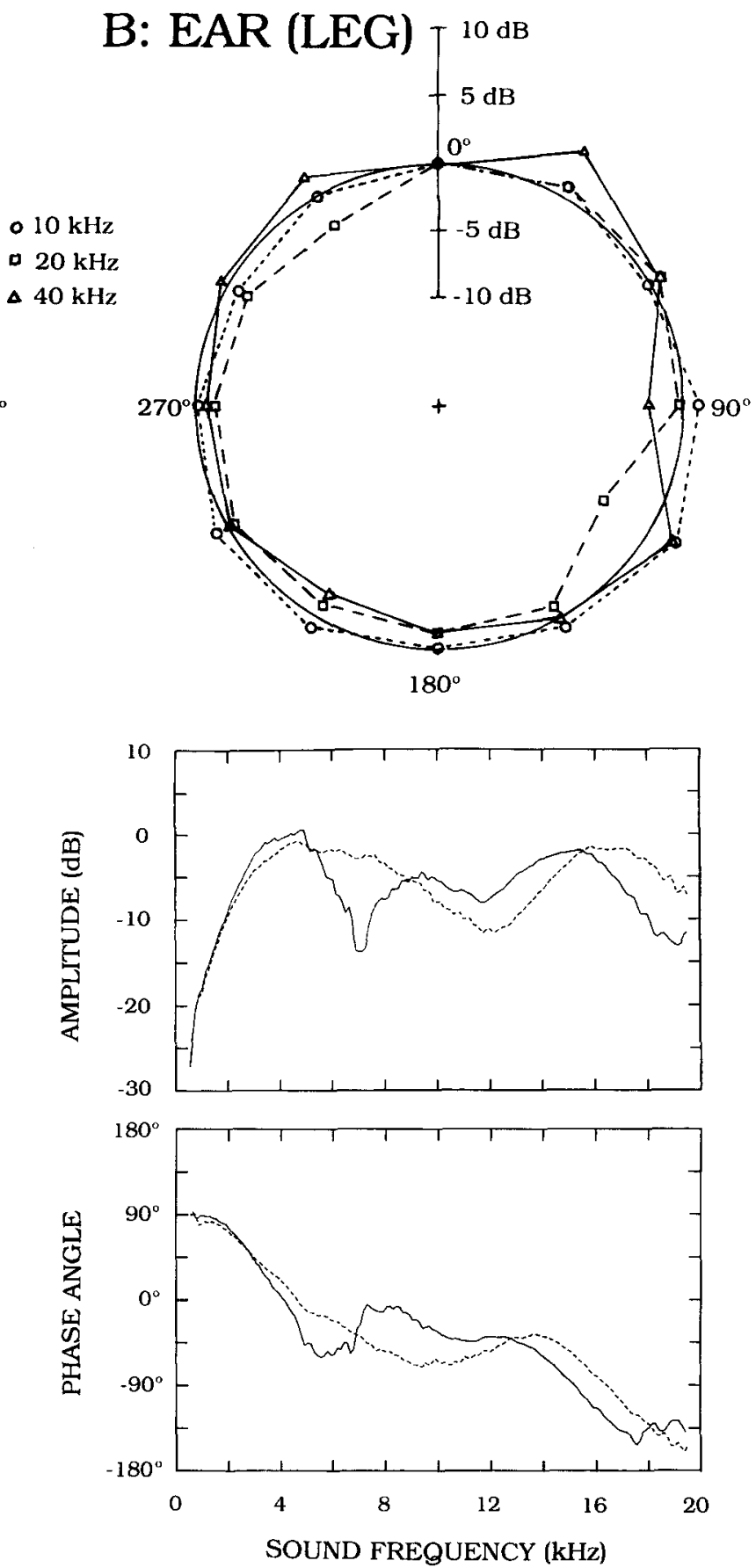

Fig. 8 Effect of the state of the acoustic trachea on the mechanics of the tympanal membrane in the cricket Gryllus bimaculatus. The tympanum is forced to vibrate by a sound pressure acting only at its external surface (cf. Fig. 1A). The transfer function obtained when the spiracle was open (solid line) differs from that obtained when the spiracle was closed with wax (dashed line). Averaged data from 4 experiments

the cricket tympanum driven exclusively by sound at its external surface, when the entrance to the acoustic trachea was blocked with wax. The change of velocity is equal to the change in impedance (since the force was constant). In this experiment it varied from a few $\mathrm{dB}$ to more than $10 \mathrm{~dB}$. 


\section{Measurements with probe microphones}

In theory, one could determine the gain of the acoustic trachea (or other sound pathways) simply by inserting a miniature microphone in the air space behind the tympanum. Physically, this is possible, given the $1 \mathrm{~mm}$ tip of the probe microphone used here. The results cannot be trusted, however, unless the input impedance of the probe microphone is sufficiently large compared to the impedance of the air space.

Unfortunately, this kind of approach has been quite popular. Furthermore, several investigators have not used commercially available probe microphones (in which the manufacturer has determined the input impedance), but home-made systems of quite unknown properties (e.g., Hill and Oldfield 1981). A recent investigation of the gain of the acoustic trachea of bushcrickets (Heinrich et al 1993) may illustrate some of the sources of error.

These investigators connected cut ends of acoustic tracheae to a custom-made probe microphone, in which the sound was guided through an opening $300 \mu \mathrm{m}$ in diameter to a hollow cone and a plastic tube of $1 \mathrm{~cm}$ diameter. A microphone in the wall of the tube measured the sound pressure inside the device, which seemed to contain c. $5 \mathrm{~cm}^{3}$ of air. The acoustic (input ?) impedance was said to be "very high" and the load of the source "small" (no figures were given). The gain of the acoustic trachea was calculated as the difference between the sound level measured with this device connected to the trachea and the sound pressure level "outside the animal" (the exact position was not specified).

The amplitude gains found by Heinrich et al were typically $10-15 \mathrm{~dB}$ at $20-30 \mathrm{kHz}$. These values were probably about $10 \mathrm{~dB}$ too low, probably because the input impedance of the home-made probe was not sufficiently high.

Acknowledgements The experimental work and the travel costs of three of us was supported by the Danish Science Research Council and the later computational work by the by the Danish National Research Foundation. We are grateful to Brain Lewis for comments on the manuscript.

\section{References}

Bailey WJ (1990) The ear of the bushcricket. In: Bailey WJ, Rentz DCF (eds) The Tettigoniidae: Biology, systematics and evolution. Springer, Berlin, pp 217-247

Heinrich R, Jatho M, Kalmring K (1993) Acoustic transmission characteristics of the tympanal tracheae of bushcrickets (Tettigoniidae). II: Comparative studies of the tracheae of seven species. J Acoust Soc Am 93: 3481-3489

Hill KG, Oldfield BP (1981) Auditory function in Tettigoniidae (Orthoptera, Ensifera). J Comp Physiol 142: 169-180

Lewis B (1983) Directional cues for auditory location. In: Lewis B (ed) Bioacoustics: a comparative approach. Academic Press, London, pp 233-260

Mason AC, Morris GK, Wall P (1991) High ultrasonic hearing and tympanal slit function in rainforest katydids. Naturwissenschaften 78: 365-367

Michelsen A, Larsen ON (1978) Biophysics of the ensiferan ear. I. Tympanal vibrations in bushcrickets (Tettigoniidae) studied with laser vibrometry. J Comp Physiol 123: 193-203

Michelsen A, Popov AV, Lewis B (1994) Physics of directional hearing in the cricket Gryllus bimaculatus J Comp Physiol A $153-164$

Stumpner A, Heller K-G (1992) Morphological and physiological differences of the auditory system in three related bushcrickets (Orthoptera: Phaneropteridae, Poecilimon). Physiol Entomol 17: $73-80$ 